\title{
Cassava Leaf Compost Influences Growth, Yield and Nutrient Uptake of Rice
}

\author{
Nazmul Huq Hawlader ${ }^{1}$, Md. Solaiman Ali Fakir ${ }^{2}$, Masum Ahmad ${ }^{3}$, \\ Habibun Nesa ${ }^{4}$, Md. Mustafizur Rahman ${ }^{4}$, Irteja Hasan ${ }^{5}$, Md. Monirul Islam ${ }^{6}$, \\ Md. Sazedul Islam ${ }^{6}$ and Md. Sagirul Islam Majumder ${ }^{7^{*}}$
}

${ }^{1}$ Department of Seed Science and Technology, Bangladesh Agricultural University, Mymensingh2202, Bangladesh.

${ }^{2}$ Department of Crop Botany, Bangladesh Agricultural University, Mymensingh-2202, Bangladesh. ${ }^{3}$ Department of Entomology, Bangladesh Agricultural University, Mymensingh-2202, Bangladesh.

${ }^{4}$ Department of Agricultural Extension (DAE), Khamarbari, Farmgate, Dhaka-1215, Bangladesh. ${ }^{5}$ Department of Geo-Information Science and Earth Observation, Patuakhali Science and Technology

${ }^{6}$ Department of Soil Science, Patuakhali Science and Technology University, Patuakhali-8602

Bangladesh.

${ }^{7}$ The United Graduate School of Agricultural Sciences, Kagoshima University, Kagoshima-890-0065,

Japan.

\section{Authors' contributions}

This work was carried out in collaboration among all authors. Author NHH designed the study, wrote the protocol, performed the experiment and prepared the first draft of the manuscript. Authors MSAF and MA supervised the study and managed the research facilities. Authors MMR, HN and IH edited the manuscript. Authors MMI and MSI performed the statistical analysis. Author MSIM checked the analytical data, statistical analysis and edited the manuscript. All authors read and approved the final manuscript.

Article Information

DOI: $10.9734 / A R R B / 2020 / v 35 i 930268$ Editor(s):

(1) Dr. Ibrahim Farah, Jackson State University, USA Reviewers:

(1) Debojyoti Roychowdhury, Techno India University, India. (2) Eliecer Cabrales Herrera, University of Córdoba, Colombia Complete Peer review History: http://www.sdiarticle4.com/review-history/58324

Original Research Article

Received 01 May 2020

Accepted 06 July 2020

Published 28 August 2020

\section{ABSTRACT}

The application of compost as alternatives to reduce the use of synthetic fertilizers is considered a feasible agricultural practice to mitigate soil degradation caused by long term application of synthetic fertilizers. Therefore, the study aimed to investigate the effect of cassava leaf compost on 
growth, yield and nutrient uptake of rice. Six treatments of two rice varieties (BRRI dhan29 and BINA dhan5) and three sources of nitrogen (Control - no nitrogen applied but residual soil $\mathrm{N}$ was $0.123 \%$, urea @ $200 \mathrm{~kg}$ ha-1 and Cassava Leaf Compost @ $10 \mathrm{t} \mathrm{ha}^{-1}$ ) were used in this study. Cassava Leaf Compost @ $10 \mathrm{t} \mathrm{ha}^{-1}$ and urea @ $200 \mathrm{~kg}$ ha-1 significantly increased effective tillers per hill, 1000-grain weight, and grain yield were 12.84 and $13.75,25.00 \mathrm{~g}$ and $24.93 \mathrm{~g}, 5.57 \mathrm{t} \mathrm{ha}^{-1}$ and $6.047 \mathrm{t} \mathrm{ha}^{-1}$, respectively. Cassava Leaf Compost @ $10 \mathrm{t} \mathrm{ha}^{-1}$ and urea @ $200 \mathrm{~kg} \mathrm{ha}^{-1}$ increased $\mathrm{N}$ uptake in root is $0.821 \%$ and $0.756 \%$, and the residual effect of compost increased $\mathrm{C}$, $\mathrm{N}, \mathrm{P}, \mathrm{K}$ and $\mathrm{S}$ availability in soil for the succeeding crop in Cassava Leaf Compost @ $10 \mathrm{t} \mathrm{ha}^{-1}$ applied plots. Carbon and $\mathrm{N}$ mineralization rates were higher than control and soils receiving recommended dose of inorganic fertilizers. Our findings suggested that compost could be used as biofertilizer to improve degraded cropland soils for sustainable agriculture.

Keywords: Cassava leaf; compost; growth; yield; nutrient uptake; soil properties.

\section{INTRODUCTION}

Rice (Oryza sativa $L$ ) is the main staple food of Bangladesh. Nitrogen $(\mathrm{N})$ is an essential macronutrient for rice and other crops. The soils in Bangladesh contain $\mathrm{N}$ with small quantities compared to the requirement for higher rice production. Sources of nitrogen are synthetic fertilizers (urea, ammonium sulphate), organic manure (cow dung, poultry manure) and compost. Urea fertilizers have been used increasingly in Bangladesh for rice production due to availability of inexpensive synthetic fertilizer. Due to application of synthetic $\mathrm{N}$ fertilizers may influence leaching loss and volatilization resulting soil quality deteriorated.

So, it is essential to find an alternative source of $\mathrm{N}$ to urea fertilizer such as organic manure, cassava leaf and other composts. Dhaincha (Sesbania rostrata) incorporated into the soil @ 17.5 ton $\mathrm{ha}^{-1}$ produced identical rice yield with that of $80 \mathrm{~kg} \mathrm{~N}^{-1}{ }^{-1}$ [1]. On the other hand the equivalent quantity of $\mathrm{N}$ would be obtained from 10-12 ton ha ${ }^{-1}$ cassava leaf compost. So, it can be assumed that cassava leaf compost is one of the most feasible alternatives to provide enough $\mathrm{N}$ in unit area of land. In cereals, the use of cassava leaf compost to increase the yield and protein content of the grain, and simultaneously reduce risks of nitrate leaching and denitrification [2], and increase number of leaves, fruit and fruit weight in vegetable [3]. In Bangladesh, cassava (Manihot esculenta Crantz) is a least known crop and sparsely grown in Madhupur, Garo Hill, Netrokona, Sherpur and Chittagong Hill Tracts areas. Experiment has revealed that the leaf dry matter of cassava plant tops contains 2.88$4.80 \% \mathrm{~N}(18-30 \%$ crude protein dry wt. basis), which consists of various essential amino acids.
Cassava leaves contain $23-31 \%$ dry matter (DM) and $23-30 \%$ crude protein (in DM) [4]. Considering the average protein content $(20 \%$ dry wt. basis) in cassava leaf, one hectare of cassava would provide $140 \mathrm{~kg}$ of protein $[5,6]$ estimated litter left by cassava is compensated for nitrogen requirement for succeeding maize crop. Like many organic compost materials cassava leaf compost is a potential reservoir of organic matter and plant nutrients. The practice improves physical, chemical and biological properties of soil and improves crop yield and nutritional value. Cassava leaf compost minimizing the use of costly purchased inorganic agro-chemicals like urea and alleviates nutrient deficiency in soil by providing great amount of nitrogen and other nutrient elements. For this reason using cassava leaves as a source of nitrogen is highly sustainable in rice cultivation. This would not occupy rice land and cassava composting can be done within 90-120 days. Organic compost is a very important method of providing plants with their nutritional requirements without having an undesirable impact on the environment [3]. There are many research information relating to canopy characters, debranching, morphology, growth, starch production of cassava is available [7-10]; there is a few report of cassava leaf compost (CLC) effect on growth and yield of rice [11].

Therefore, to achieve a potential yield of rice, adequate nitrogen, phosphorus, and potassium fertilization is essential. No study on the effect of cassava leaf compost on growth, yield and nutrient uptake of rice has been done in Bangladesh. The findings will help to facilitate the potential of cassava leaf compost in the rice field before being introduced to local farmers. The objectives of this study were to (i) evaluate the growth and yield of rice and (ii) to determine the 
nutrient uptake of rice cultivated in cassava leaf compost treated soil.

\section{MATERIALS AND METHODS}

The research was conducted at the Field of Crop Botany department, Bangladesh Agricultural University (BAU), Mymensingh $\left(24.75^{\circ} \mathrm{N}\right.$ and $90.50^{\circ} \mathrm{E}$ ) to evaluate the effect of cassava leaf compost as an alternatives in rice cultivated in non-calcareous dark grey floodplain soil under the agro-ecological Zone of the Brahmaputra Floodplain.

\subsection{Experimental Design}

This factorial experiment was laid out in a Randomized Complete Block Design (RCBD) with three replications. The factors and treatments included in the experiments were as follows-Factor A: Variety i. BRRI dhan29 $\left(\mathrm{V}_{1}\right)$ ii. BINA dhan5 $\left(\mathrm{V}_{2}\right)$ Factor B: Sources of Nitrogen i. $\mathrm{C}=$ Control (No Nitrogen applied but residual soil $\mathrm{N}$ was $0.123 \%)\left(\mathrm{T}_{1}\right)$ ii. $\mathrm{U}=$ Urea Fertilizer@ 200kg/ha $\left(T_{2}\right)$ iii. CLC = Cassava Leaf Compost @ $10 \mathrm{t} / \mathrm{ha}\left(\mathrm{T}_{3}\right)$. A $25 \mathrm{~cm}$ layer of composting material (dried cassava leaves) was placed over the ground surface. This layer consisted with three sub layers- bottom $(15 \mathrm{~cm}$ cassava leaves and twigs), middle (5 cm cowdung) and top (2.5$5 \mathrm{~cm}$ clay soil). These steps were repeated until the compost heap reached to $1.25 \mathrm{~m}$ height. Urea fertilizer (100 $\mathrm{g}$ for each layer) was added to increase the microbial activities for decomposition. The heap was covered with polythene sheet to protect from rainfall. After three months the compost was ready for use (Fig. 1). Cassava leaf compost was appliedat two splits dose: half at land preparation and the remaining half at 60 days after transplanting (panicle initiation stage). Urea fertilizer was applied into three equal splits, $1 / 3^{\text {rd }}$ was applied at 15 DAT, and second $1 / 3^{\text {rd }}$ was applied at 40 DAT (active tillering stage) and remaining $1 / 3^{\text {rd }}$ at 60 DAT (panicle initiation stage).

\subsection{Laboratory Analysis}

Soil $\mathrm{pH}$ was determined with a glass electrode $\mathrm{pH}$ meter (WTW pH 522; Germany) [12]. The organic carbon was determined using the Walkley and Black wet oxidation method [13].
Nitrogen of the soil samples was determined by Kjeldahl method [14]. Available phosphorus was determined by the Olsen method [15] using spectrophotometer at $710 \mathrm{~nm}$ wave length. Potassium and sodium were determined from the aliquot separately with the help of flame emission spectrophotometer (Model: JENWAY-PFP7) at $589 \mathrm{~nm}$ and $768 \mathrm{~nm}$ for sodium and potassium, respectively [16]. Available $S$ content was determined by turbidimetric method [17]. Exchangeable cations are extracted from the soil using an extracting solution (1 NNH4OAc) at $\mathrm{pH}$ 7.0. The extracted solution is then analyzed by AA (atomic absorption) for the soil cations [18]. The properties of experimental soil and cassava leaf compost have been mentioned in Table 1.

\subsection{Data Measurement}

Data on the following parameters; Plant height $(\mathrm{cm})$, chlorophyll content (SPAD reading), panicle length $(\mathrm{cm})$, effective tillers hill $^{-1}$ (no.), total tillers hill $^{-1}$ (no.), spikelets panicle ${ }^{-1}$ (no.), total spikelets panicle $e^{-1}$ (no.), \% fertile spikelets, 1000- grain weight $(\mathrm{g})$, grain yield $\left(\mathrm{t} \mathrm{ha}^{-1}\right)$, insect infestation (YSB) plant plot ${ }^{-1}$, root weight (g), root length and width $(\mathrm{cm})$ and harvest index were recorded. Plant height, total number of tillers hill ${ }^{-1}$ and chlorophyll content were recorded at three growth stages viz., panicle initiation stage (65 DAP), grain filling stage (90 DAP) and maturity stage (120 DAP). Grains and straw data collected from the central $1 \mathrm{~m}^{2}$ area and those from sample hills of each plot were sun dried and weighted carefully. Dry weight of grains of each plots was converted into grain yield at $14 \%$ moisture content through air drying and expressed in ton $\mathrm{ha}^{-1}$.

$$
\begin{aligned}
& \text { Biological yield }=\text { Grain yield }+ \text { Straw yield. } \\
& \text { Harvest index }=\frac{\text { Grain yield } / \text { plot }}{\text { Biological yield } / \text { plot }} \times 100
\end{aligned}
$$

\subsection{Statistical Analysis}

All the collected data were analyzed following the analysis of variance (ANOVA) technique and mean difference were adjusted by Duncan's Multiple Range Test (DMRT) [19] using MSTAT program. 
Table 1. The experimental field soil and cassava leaf compost properties are listed given bellow

\begin{tabular}{lll}
\hline \multicolumn{1}{c}{ The experimental field soil } & & Cassava leaf compost \\
\hline Soil pH & 7.2 & 7.5 \\
Total nitrogen $(\mathrm{N})$ & $0.12 \%$ & $1542 \%$ \\
Organic carbon $(\mathrm{C})$ & $1.14 \%$ & $17.40 \%$ \\
Available phosphorus $(\mathrm{P})$ & $7.26 \mathrm{ppm}$ & $228.8 \mathrm{ppm}$ \\
Exchangeable K (me $/ 100 \mathrm{~g}$ soil) & 48.35 & $2567.9 \mathrm{ppm}$ \\
Exchangeable cation $(\mathrm{mS} / \mathrm{cm})$ & 1.47 & 2.63 \\
Available Sulphur $(\mathrm{S})$ & $82.72 \mathrm{ppm}$ & $189.4 \mathrm{ppm}$ \\
Available Sodium $(\mathrm{Na})$ & $104.0 \mathrm{ppm}$ & $1200.0 \mathrm{ppm}$ \\
\hline
\end{tabular}
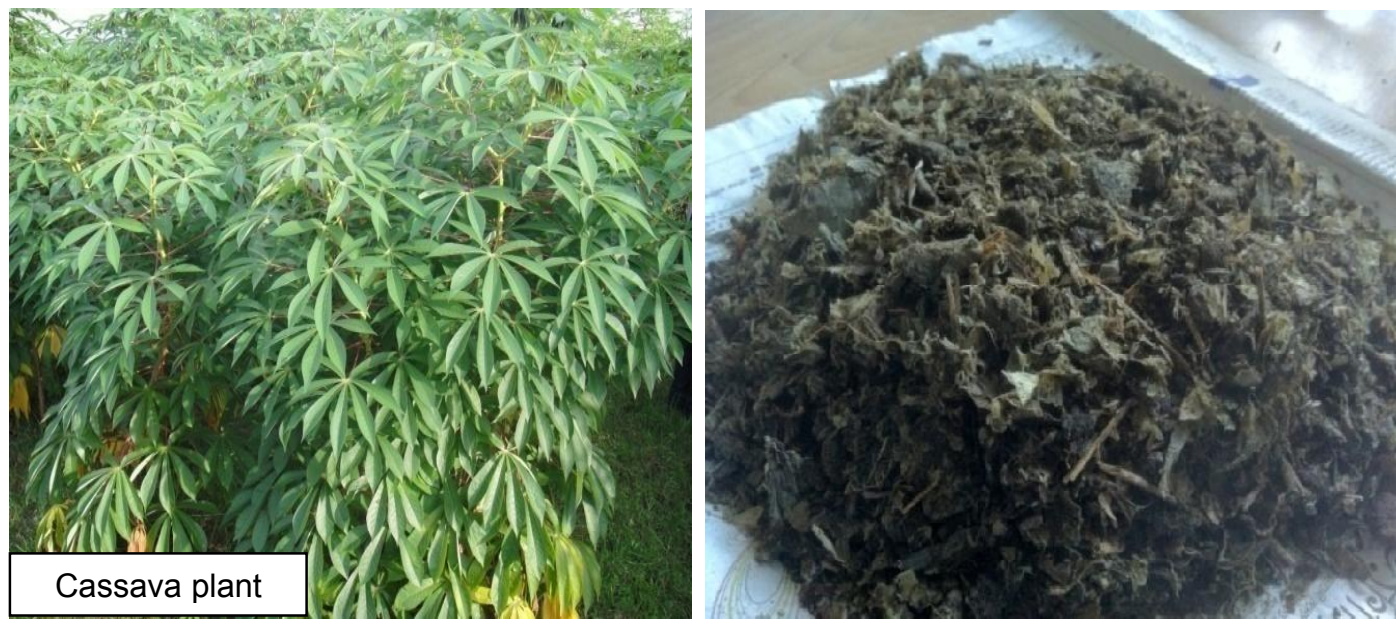

Fig. 1. Showing preparation of cassava leaf compost

\section{RESULTS AND DISCUSSION}

\subsection{Effect on Growth Parameter}

At panicle initiation stage, plants were shorter in control plot $(62.5 \mathrm{~cm})$ than urea and CLC treated plots (average of $68.9 \mathrm{~cm}$ ). In BRRI dhan29, urea and CLC treated plant height were similar and higher than control. In BINA dhan5, it was significantly smaller in control plot $(69.0 \mathrm{~cm})$ than in urea and CLC treated plots (average of $75.9 \mathrm{~cm}$ ) (Table 2). At grain filling stage, treatments had significant effect on plant height. Plants were shorter in control plot $(83.6 \mathrm{~cm}$ ) compared to urea and CLC treated plots (average of $92.2 \mathrm{~cm}$ ). Variety showed significant effect on plant height in which BINA dhan5 was taller $(98.6 \mathrm{~cm})$ than BRRI dhan29 $(80.1 \mathrm{~cm})$. The interaction had no significant influences on plant height.

At maturity stage, interaction showed no significant effect on plant height. However genetic variation was observed. The taller plant was obtained from BINA dhan5 $(104.4 \mathrm{~cm})$ than BRRI dhan29 $(84.4 \mathrm{~cm})$. Similar result was reported by some researchers [20]. At maturity stage the number of tillers hill ${ }^{-1}(15.0 \mathrm{~cm}$ and $15.0 \mathrm{~cm}$ for urea and CLC, respectively) was higher than control. The lowest number of tillers hill $^{-1}(12.5)$ at grain filling stage was found in control.

At grain filling stage the higher chlorophyll content (40.6) was observed from application of urea at recommended dose of $200 \mathrm{~kg} \mathrm{ha}^{-1}$. Chlorophyll content was affected by variety. The highest chlorophyll content (38.8) was obtained from BRRI dhan29 at grain filling stage. Lower chlorophyll content (20.1) was obtained from BINA dhan5 at maturity stage. Chlorophyll content was increased between panicle initiation and grain filling stage followed by a decline at maturity stage in all treatments. The order of infestation was as follows: BINA dhan $5 \times$ Urea (19.1) >BRRI dhan29 $\times$ urea (15.3) >BRRI dhan29 $\times$ leaf compost (11.1) >BRRI dhan29 $\times$ control and BINA dhan5 $\times$ Leaf compost (av. 7.6) $>$ BINA dhan5 $\times$ control (7.00) (Table 2). 


\subsection{Effect on Morphological and Yield Attributes at Maturity}

The higher panicle length $(25.4 \mathrm{~cm})$ was obtained from cassava leaf compost treated plot. The shorter panicle length was obtained from without any application of nitrogen. Incorporation of cassava leaf compost (CLC) had significant effect on number of effective tillers hill $^{-1}$. The results indicated that the higher number of effective tillers hill $^{-1}$ (13.7) was produced by the application of recommended dose of urea @ 200 kg/ha and cassava leaf compost @ 10 t/ha (12.8) compared to control plant (11.6). Similar results was found by others [21] who reported that inorganic manure increased productive tillers and plant height at harvest. Genetic differences in number of effective tillers hill $^{-1}$ was insignificant for both varieties with BRRIdhan29 produced increased number of effective tillers (13.0) than BINAdhan5 (12.4). This result was in line with others [22,23]. Significant difference was observed when different treatments were applied in the production of total tillers hill $^{-1}$. Result presented in Table 3 shows that the total number of tillers hill $^{-1}(15.07)$ were highest when applied cassava leaf compost at $10 \mathrm{t} \mathrm{ha}^{-1}$. The fewest tillers hill ${ }^{-1}$ (12.42) was obtained in control plot. It was found from this study that urea and cassava leaf compost produced the highest number of spikelet's panicle ${ }^{-1}$ (145.8 and 148.5 for urea and CLC respectively). The fewest (115.9) was obtained from control. Number of spikelet's panicle $^{-1}$ was significantly influenced by the variety. Numerically, the higher number of spikelet's panicle $^{-1}$ (138.0) was produced by BINA dhan5. Fewer spikelets'panicle ${ }^{-1}$ (135.2) was produced by BRRI dhan29. But number of total spikelets panicle ${ }^{-1}$ was not significantly influenced by the variety. Urea and cassava leaf compost the highest number of total spikelets panicle $^{-1}$ (161.9 and 160.6) respectively. The highest $\%$ fertile spikelets panicle ${ }^{-1}$ (92.79\%) were found from the recommended dose of cassava leaf compost and the lowest (84.04\%) was obtained from without any application of nitrogen (Table 3).

Nitrogen treatment exhibited significant effect on grain yield. The results showed that recommended dose of urea @ 200 kg/ha gave the highest grain yield (6.04 t/ha) which was somewhat lower (5.57 t/ha) with cassava leaf compost @ 10 t/ha. The lowest grain yield (4.558 t/ha) was obtained from control plant. The highest grain yield might be the cumulative effect of increased number of effective tillers hill ${ }^{-1}$, greater spikelet's panicle ${ }^{-1}$ and heavier 100-grain weight. The result was in conformity with other researchers [24,25]. Grain yield was significantly differed variety BINA dhan5 produced more yield than BRRI dhan29. Grain yield was significantly influenced by interaction effect. The highest grain yield (6.233 t/ha) was obtained from recommended dose of urea $\times$ BINAdhan5 followed by cassava leaf compost $\times$ BINAdhan5 (6.01 t/ha). The lowest grain yield (4.51 t/ha) was obtained from control $\times$ BRRIdhan29 (Table 3).

\subsection{Effect on Dry Matter Production and Partitioning}

The higher grain weight (12.15g and 11.28g) were obtained from cassava leaf compost and urea application (Table 4). The lower grain weight $(9.30 \mathrm{~g})$ was obtained from without any application of nitrogen. Interaction showed non-significant effect on grain weight of Boro rice. The effect of nitrogen exerted a significant effect on harvest index. Highest harvest index was at urea and cassava leaf compost treated plots (average of $36.14 \%$ ) than control (33.78 \%). Harvest index was significant at $1 \%$ level of probability due to variety (Table 4). Result shows that the higher harvest index $(36.37 \%)$ was recorded from BRRI dhan29 than BINA dhan5 (34.34\%). Interaction effect on harvest index was found nonsignificant.

\subsection{Effect on Root Growth and Development}

The highest fresh weight $15.6 \mathrm{~g}$ was obtained from cassava leaf compost. The lowest root weight $(10.6 \mathrm{~g})$ was obtained without any application of nitrogen. Nitrogen treatment exhibited significant effect on root dry matter production. The result showed that higher root dry weight was found in cassava leaf compost $(13.6 \mathrm{~g})$ than control $(9.6 \mathrm{~g})$. Highest in cassava leaf compost $(13.4 \mathrm{~g})$ and lowest in urea applied plot $(10.4 \mathrm{~g})$. Highest root length $(23.7 \mathrm{~cm})$ was obtained from cassava leaf compost application. The shorter root length was obtained from without any application of nitrogen $(21.0 \mathrm{~cm})$. Highest root canopy $(10.5 \mathrm{~cm})$ was in cassava leaf compost application and lowest $(9.5 \mathrm{~cm})$ in control with intermediate $(9.7 \mathrm{~cm})$ in urea application (Table 5). 
Hawlader et al.; ARRB, 35(9): 23-33, 2020; Article no.ARRB.58324

Table 2. Effect of urea and cassava leaf compost on plant height, tiller and chlorophyll production, and borer infestation at three growth stages of two rice varieties

\begin{tabular}{|c|c|c|c|c|c|c|c|c|c|c|}
\hline \multirow[t]{2}{*}{ Treatments } & \multicolumn{3}{|c|}{ Plant height $(\mathrm{cm})$} & \multicolumn{3}{|c|}{ Total number of tillers hill $^{-1}$} & \multicolumn{3}{|c|}{ Chlorophyll content (SPAD reading) } & \multirow[b]{2}{*}{$\begin{array}{l}\text { Infestation } \\
\text { by yellow } \\
\text { rice stem } \\
\text { borer } \\
\text { (YSB) }\end{array}$} \\
\hline & $\begin{array}{l}\text { Panicle } \\
\text { initiation } \\
\text { stage }\end{array}$ & $\begin{array}{l}\text { Grain } \\
\text { filling } \\
\text { stage }\end{array}$ & $\begin{array}{l}\text { Maturity } \\
\text { stage }\end{array}$ & $\begin{array}{l}\text { Panicle } \\
\text { initiation } \\
\text { stage }\end{array}$ & $\begin{array}{l}\text { Grain } \\
\text { filling } \\
\text { stage }\end{array}$ & $\begin{array}{l}\text { Maturity } \\
\text { stage }\end{array}$ & $\begin{array}{l}\text { Panicle } \\
\text { initiation } \\
\text { stage }\end{array}$ & $\begin{array}{l}\text { Grain filling } \\
\text { stage }\end{array}$ & $\begin{array}{l}\text { Maturity } \\
\text { stage }\end{array}$ & \\
\hline \multicolumn{11}{|l|}{ Nitrogen (N) } \\
\hline Control $(\mathrm{C})$ & $62.5^{\mathrm{c}}$ & $83.67^{c}$ & $90.96^{b}$ & $12.70^{\mathrm{C}}$ & $12.52^{\mathrm{C}}$ & $12.42^{b}$ & $31.75^{\mathrm{c}}$ & $35.38^{c}$ & $21.88^{c}$ & $7.33^{\mathrm{C}}$ \\
\hline Urea $(U)$ & $71.33^{\mathrm{a}}$ & $94.42^{a}$ & $96.95^{\mathrm{a}}$ & $16.16^{\mathrm{b}}$ & $15.5^{\mathrm{b}}$ & $15.05^{a}$ & $37.72^{a}$ & $40.65^{a}$ & $25.92^{\mathrm{a}}$ & $17.25^{\mathrm{a}}$ \\
\hline Leaf compost (LC) & $66.53^{\mathrm{b}}$ & $90.09^{b}$ & $95.41^{a}$ & $16.38^{a}$ & $15.64^{\mathrm{a}}$ & $15.07^{\mathrm{a}}$ & $36.15^{\mathrm{b}}$ & $38.81^{\mathrm{b}}$ & $24.45^{\mathrm{b}}$ & $9.66^{\mathrm{b}}$ \\
\hline \multicolumn{11}{|l|}{ Variety (V) } \\
\hline BRRI dhan29 $\left(\mathrm{V}_{1}\right)$ & $59.93^{b}$ & $80.12^{b}$ & $84.43^{\mathrm{b}}$ & $15.14^{\mathrm{a}}$ & $14.91^{\mathrm{a}}$ & $14.32^{\mathrm{a}}$ & $34.76^{\mathrm{b}}$ & $38.85^{\mathrm{a}}$ & $28.01^{a}$ & $11.55^{\mathrm{a}}$ \\
\hline BINA dhan5 $\left(V_{2}\right)$ & $73.65^{\mathrm{a}}$ & $98.66^{a}$ & $104.4^{\mathrm{a}}$ & $15.02^{\mathrm{b}}$ & $14.22^{\mathrm{b}}$ & $14.04^{\mathrm{b}}$ & $35.66^{\mathrm{a}}$ & $37.71^{\mathrm{b}}$ & $20.16^{\mathrm{b}}$ & $11.28^{\mathrm{b}}$ \\
\hline \multicolumn{11}{|l|}{ Interaction $(\mathrm{N} \times \mathrm{V})$} \\
\hline $\mathrm{V}_{1} \mathrm{C}$ & $55.9^{\dagger}$ & $75.1^{\dagger}$ & $82.8^{\mathrm{d}}$ & $12.9^{\mathrm{d}}$ & $12.7^{\mathrm{d}}$ & $12.6^{\mathrm{d}}$ & $30.9^{e}$ & $36.0^{\mathrm{e}}$ & $24.7^{\mathrm{C}}$ & $7.6^{\mathrm{d}}$ \\
\hline $\mathrm{V}_{1} \mathrm{U}$ & $63.3^{d}$ & $84.3^{d}$ & $85.6^{\mathrm{C}}$ & $16.0^{c}$ & $15.9^{\mathrm{a}}$ & $15.2^{\mathrm{a}}$ & $37.2^{\mathrm{b}}$ & $41.2^{a}$ & $30.6^{a}$ & $15.3^{b}$ \\
\hline $\mathrm{V}_{1} \mathrm{LC}$ & $60.5^{\mathrm{e}}$ & $80.9^{e}$ & $84.7^{\text {cd }}$ & $16.5^{\mathrm{a}}$ & $16.0^{\mathrm{a}}$ & $15.1^{\mathrm{a}}$ & $36.0^{c}$ & $39.2^{c}$ & $28.6^{\mathrm{b}}$ & $11.6^{c}$ \\
\hline $\mathrm{V}_{2} \mathrm{C}$ & $69.0^{\mathrm{C}}$ & $92.2^{\mathrm{C}}$ & $99.0^{\mathrm{b}}$ & $12.5^{\mathrm{e}}$ & $12.3^{\mathrm{e}}$ & $12.2^{\mathrm{e}}$ & $32.5^{\mathrm{d}}$ & $34.7^{\dagger}$ & $19.0^{\dagger}$ & $7.0^{\mathrm{e}}$ \\
\hline $\mathrm{V}_{2} \mathrm{U}$ & $79.3^{\mathrm{a}}$ & $104.5^{\mathrm{a}}$ & $108.2^{a}$ & $16.3^{\mathrm{b}}$ & $15.1^{\mathrm{c}}$ & $14.9^{c}$ & $38.1^{a}$ & $40.0^{b}$ & $21.1^{\mathrm{d}}$ & $19.1^{\mathrm{a}}$ \\
\hline $\mathrm{V}_{2} \mathrm{LC}$ & $72.5^{\mathrm{b}}$ & $99.2^{\mathrm{b}}$ & $106.1^{\mathrm{a}}$ & $16.2^{\mathrm{b}}$ & $15.2^{\mathrm{b}}$ & $15.0^{\mathrm{b}}$ & $36.2^{c}$ & $38.3^{d}$ & $20.2^{\mathrm{e}}$ & $7.6^{\mathrm{d}}$ \\
\hline $\mathrm{N}$ levels $(\mathrm{A})$ & $<0.01$ & $<0.01$ & $<0.01$ & $<0.01$ & $<0.01$ & $<0.01$ & $<0.01$ & $<0.01$ & $<0.01$ & $<0.01$ \\
\hline Variety (B) & $<0.01$ & $<0.01$ & $<0.01$ & $<0.01$ & $<0.01$ & $<0.01$ & $<0.01$ & $<0.01$ & $<0.01$ & $<0.01$ \\
\hline$A \times B$ & $<0.01$ & $<0.01$ & $<0.01$ & $<0.01$ & $<0.01$ & $<0.01$ & $<0.01$ & $<0.01$ & $<0.01$ & $<0.01$ \\
\hline
\end{tabular}

In each column, under a particular treatment, figure followed by dissimilar letter (s) differ significantly at $P \leq 0.05$ by DMRT 
Hawlader et al.; ARRB, 35(9): 23-33, 2020; Article no.ARRB.58324

Table 3. Effect of urea and cassava leaf compost on morphological and yield attributes at maturity (142 DAS) in two rice varieties

\begin{tabular}{|c|c|c|c|c|c|c|c|c|c|c|}
\hline Treatments & $\begin{array}{l}\text { Plant } \\
\text { height }(\mathrm{cm})\end{array}$ & $\begin{array}{l}\text { Panicle } \\
\text { length } \\
(\mathrm{cm})\end{array}$ & $\begin{array}{l}\text { Effective } \\
\text { tillers } \\
\text { (No.) }\end{array}$ & $\begin{array}{l}\text { Total } \\
\text { tillers } \\
\text { (No.) }\end{array}$ & $\begin{array}{l}\text { Filled } \\
\text { spikelet's } \\
\text { (No.) }\end{array}$ & $\begin{array}{l}\text { Total } \\
\text { spikelet }\end{array}$ & $\begin{array}{l}\text { \% Fertile } \\
\text { spikelet }\end{array}$ & $\begin{array}{l}1000 \\
\text { grain wt. } \\
\text { (g) }\end{array}$ & $\begin{array}{l}\text { Straw yield } \\
\text { (t/ha) }\end{array}$ & $\begin{array}{l}\text { Grain } \\
\text { yield } \\
\text { (t/ha) }\end{array}$ \\
\hline \multicolumn{11}{|l|}{ Nitrogen(N) } \\
\hline Control (C) & $90.96^{b}$ & $23.06^{b}$ & $11.65^{c}$ & $12.42^{b}$ & $115.9^{\mathrm{C}}$ & $138.0^{c}$ & $84.04^{c}$ & $23.26^{b}$ & $8.98^{\mathrm{C}}$ & $4.55^{\mathrm{C}}$ \\
\hline Urea (U) & $96.83^{a}$ & $25.18^{a}$ & $13.75^{a}$ & $15.05^{\mathrm{a}}$ & $145.8^{\mathrm{b}}$ & $161.9^{a}$ & $90.04^{b}$ & $24.93^{a}$ & $10.81^{a}$ & $6.04^{a}$ \\
\hline Leaf compost (LC) & $95.41^{a}$ & $25.48^{\mathrm{a}}$ & $12.84^{\mathrm{b}}$ & $15.07^{\mathrm{a}}$ & $148.5^{\mathrm{a}}$ & $160.9^{b}$ & $92.79^{a}$ & $25.01^{a}$ & $9.78^{\mathrm{b}}$ & $5.57^{\mathrm{b}}$ \\
\hline \multicolumn{11}{|l|}{ Variety (V) } \\
\hline BRRI dhan29 $\left(\mathrm{V}_{1}\right)$ & $84.43^{b}$ & $24.53^{\mathrm{a}}$ & $13.01^{\mathrm{a}}$ & $14.32^{\mathrm{a}}$ & $135.2^{b}$ & $153.1^{b}$ & $88.22^{b}$ & $23.83^{b}$ & $9.03^{b}$ & $5.16^{b}$ \\
\hline BINA dhan5 $\left(\mathrm{V}_{2}\right)$ & $104.4^{\mathrm{a}}$ & $24.62^{\mathrm{a}}$ & $12.48^{b}$ & $14.04^{\mathrm{b}}$ & $138.3^{\mathrm{a}}$ & $154.1^{a}$ & $89.69^{a}$ & $24.97^{a}$ & $10.67^{a}$ & $5.61^{\mathrm{a}}$ \\
\hline \multicolumn{11}{|l|}{ Interaction $(\mathbf{N} \times \mathbf{V})$} \\
\hline $\mathrm{V}_{1} \mathrm{C}$ & $82.8^{d}$ & $22.4^{\mathrm{d}}$ & $11.5^{\mathrm{d}}$ & $12.6^{\mathrm{d}}$ & $116.4^{\mathrm{C}}$ & $141.4^{\mathrm{e}}$ & $82.32^{d}$ & $22.7^{\mathrm{a}}$ & $8.2^{\dagger}$ & $4.5^{\mathrm{d}}$ \\
\hline $\mathrm{V}_{1} \mathrm{U}$ & $85.6^{\mathrm{C}}$ & $25.1^{\mathrm{b}}$ & $14.2^{\mathrm{a}}$ & $15.2^{\mathrm{a}}$ & $141.4^{\mathrm{b}}$ & $158.0^{d}$ & $89.57^{\mathrm{b}}$ & $24.3^{\mathrm{a}}$ & $10.3^{c}$ & $5.8^{\mathrm{b}}$ \\
\hline$V_{1} L C$ & $84.7^{\mathrm{cd}}$ & $25.6^{a}$ & $13.1^{b}$ & $15.1^{a}$ & $147.8^{\mathrm{a}}$ & $160.0^{C}$ & $92.78^{a}$ & $24.4^{a}$ & $8.5^{\mathrm{e}}$ & $5.1^{\mathrm{c}}$ \\
\hline $\mathrm{V}_{2} \mathrm{C}$ & $99.0^{\mathrm{b}}$ & $23.6^{\mathrm{c}}$ & $11.7^{\mathrm{d}}$ & $12.2^{\mathrm{e}}$ & $115.5^{\mathrm{C}}$ & $134.7^{\dagger}$ & $85.76^{\mathrm{C}}$ & $23.7^{\mathrm{a}}$ & $9.7^{\mathrm{d}}$ & $4.6^{d}$ \\
\hline $\mathrm{V}_{2} \mathrm{U}$ & $108.0^{a}$ & $25.1^{\mathrm{ab}}$ & $13.2^{b}$ & $14.9^{c}$ & $150.2^{a}$ & $165.9^{a}$ & $90.52^{b}$ & $25.5^{\mathrm{a}}$ & $11.2^{\mathrm{a}}$ & $6.2^{a}$ \\
\hline$V_{2} L C$ & $106.1^{a}$ & $25.0^{\mathrm{b}}$ & $12.5^{\mathrm{c}}$ & $15.0^{\mathrm{b}}$ & $149.1^{a}$ & $161.8^{b}$ & $92.7^{\mathrm{a}}$ & $25.6^{a}$ & $11.0^{\mathrm{b}}$ & $6.0^{\mathrm{b}}$ \\
\hline$N$ levels $(A)$ & $<0.01$ & $<0.01$ & $<0.01$ & $<0.01$ & $<0.01$ & $<0.01$ & $<0.01$ & $<0.01$ & $<0.01$ & $<0.01$ \\
\hline Variety (B) & $<0.01$ & 0.03 & $<0.01$ & $<0.01$ & $<0.01$ & $<0.01$ & $<0.01$ & $<0.01$ & $<0.01$ & $<0.01$ \\
\hline$A \times B$ & $<0.01$ & $<0.01$ & $<0.01$ & $<0.01$ & $<0.01$ & $<0.01$ & $<0.01$ & 0.04 & $<0.01$ & $<0.01$ \\
\hline
\end{tabular}

In each column, under a particular treatment, figure followed by dissimilar letter (s) differ significantly at $P \leq 0.05$ by DMRT 


\subsection{Nitrogen Uptake by Grain, Stem and Root}

Nitrogen uptake in grain was higher in urea $(1.8 \%)$ followed by the cassava leaf compost $(1.5 \%)$ and control $(1.2 \%)$ plot. Varietal differences existed; BRRI dhan29 had the higher $(1.6 \%)$ than BINA dhan5 (1.5\%). Interaction showed non-significant effect in nitrogen uptake (\%). Nitrogen uptake in stem was higher in urea $(1.2 \%)$ than cassava leaf compost $(1.0 \%)$ and control $(0.5 \%)$. Varietal difference existed; BRRI dhan29 had the higher $(1.1 \%)$ than BINA dhan5 (0.7\%). Interaction effect on nitrogen uptake was significant where CLC showed always higher performance (Table 6).

Nitrogen uptake in root was higher in cassava leaf compost $(0.8 \%)$ than urea $(0.7 \%)$ and control (0.4\%). Varietal difference existed; BINA dhan5 had the higher $(0.6 \%)$. than BRRI dhan29 $(0.6 \%)$. Interaction effect on nitrogen uptake was significant where CLC showed always higher performance (Table 6).

\subsection{Residual Effect of Cassava leaf Compost and Fertilizers on Soil}

The different levels of nitrogen treatments applied on the rice experiments had residual effect in the soil of rice after harvesting. Consequently, the percent organic carbon of that soil before rice transplanting was low compared with CLC soil after harvesting rice due to residual effect of cassava leaf compost (Table 7). Considerable, low amount of nitrogen $(\mathrm{N})$, phosphorus $(\mathrm{P})$, potassium $(\mathrm{K})$, and sulphur $(\mathrm{S})$ were found in soil before transplanting rice. But the amount of this nutrient element was increased after harvesting of rice crop due to residual effect of cassava leaf compost. The residual effect on soil properties especially $\mathrm{C}, \mathrm{N}$, $\mathrm{K}$ and $\mathrm{S}$ availability was much better in the CLC than in the urea treated plots (Table 7 ). In case in organic $\mathrm{C}(17.4 \%)$, available $\mathrm{N}(1.5 \%)$, $\mathrm{P}$ (228.7 ppm), K (2567.9 ppm) and S (189.4 ppm) was observed higher in CLC than urea application (Table 7). It may be concluded that cassava leaf compost appeared to increase $20 \%$ grain yield and improved soil health.

Table 4. Effect of urea and cassava leaf compost on dry matter production and partioning per 5 tillers at maturity (142 DAS) of two rice varieties

\begin{tabular}{llllll}
\hline Treatments & $\begin{array}{l}\text { Filled grain } \\
\text { weight / 5- } \\
\text { tillers (g) }\end{array}$ & $\begin{array}{l}\text { Unfilled grain } \\
\text { wt. / 5-tillers (g) }\end{array}$ & $\begin{array}{l}\text { Weight of } \\
\text { Leaf sheath } \\
(\mathbf{g})\end{array}$ & $\begin{array}{l}\text { Wt. of stem+ } \\
\text { leaf sheath+ } \\
\text { spikelet } \mathbf{( g )}\end{array}$ & $\begin{array}{l}\text { Harvest } \\
\text { index } \\
(\%)\end{array}$ \\
\hline Nitrogen (N) & & & & & \\
\hline Control (C) & $9.30^{\mathrm{c}}$ & $0.51^{\mathrm{c}}$ & $1.87^{\mathrm{c}}$ & $6.40^{\mathrm{c}}$ & $33.78^{\mathrm{c}}$ \\
Urea (U) & $11.28^{\mathrm{b}}$ & $0.88^{\mathrm{a}}$ & $3.15^{\mathrm{a}}$ & $8.79^{\mathrm{a}}$ & $35.87^{\mathrm{b}}$ \\
Leaf compos (LC) & $12.15^{\mathrm{a}}$ & $0.62^{\mathrm{b}}$ & $2.53^{\mathrm{b}}$ & $7.83^{\mathrm{b}}$ & $36.42^{\mathrm{a}}$ \\
\hline Variety (V) & & & & & \\
\hline BRRI dhan29 $\left(\mathrm{V}_{1}\right)$ & $10.17^{\mathrm{b}}$ & $0.65^{\mathrm{b}}$ & $1.83^{\mathrm{b}}$ & $6.09^{\mathrm{b}}$ & $36.37^{\mathrm{a}}$ \\
BINA dhan5 $\left(\mathrm{V}_{2}\right)$ & $11.65^{\mathrm{a}}$ & $0.69^{\mathrm{a}}$ & $3.20^{\mathrm{a}}$ & $9.25^{\mathrm{a}}$ & $34.34^{\mathrm{b}}$ \\
\hline Interaction (N $\times \mathbf{V})$ & & & & & \\
\hline $\mathrm{V}_{1} \mathrm{C}$ & $8.2^{\mathrm{e}}$ & $0.4^{\mathrm{e}}$ & $1.3^{\mathrm{f}}$ & $5.0^{\mathrm{f}}$ & $35.4^{\mathrm{d}}$ \\
$\mathrm{V}_{1} \mathrm{U}$ & $10.8^{\mathrm{c}}$ & $0.8^{\mathrm{b}}$ & $2.1^{\mathrm{d}}$ & $6.9^{\mathrm{d}}$ & $36.1^{\mathrm{b}}$ \\
$\mathrm{V}_{1}$ LC & $11.4^{\mathrm{b}}$ & $0.7^{\mathrm{c}}$ & $2.0^{\mathrm{e}}$ & $6.3^{\mathrm{e}}$ & $37.5^{\mathrm{a}}$ \\
$\mathrm{V}_{2} \mathrm{C}$ & $10.3^{\mathrm{d}}$ & $0.5^{\mathrm{d}}$ & $2.4^{\mathrm{c}}$ & $7.8^{\mathrm{c}}$ & $32.0^{\mathrm{e}}$ \\
$\mathrm{V}_{2} \mathrm{U}$ & $11.7^{\mathrm{b}}$ & $0.9^{\mathrm{a}}$ & $4.1^{\mathrm{a}}$ & $10.6^{\mathrm{a}}$ & $35.6^{\mathrm{c}}$ \\
$\mathrm{V}_{2}$ LC & $12.8^{\mathrm{a}}$ & $0.5^{\mathrm{d}}$ & $3.0 \mathrm{~b}$ & $9.3^{\mathrm{b}}$ & $35.3^{\mathrm{e}}$ \\
$\mathrm{N}$ levels (A) & $<0.01$ & $<0.01$ & $<0.01$ & $<0.01$ & $<0.01$ \\
Variety (B) & $<0.01$ & $<0.01$ & $<0.01$ & $<0.01$ & 0.05 \\
A x B & $<0.01$ & $<0.01$ & $<0.01$ & $<0.01$ & $<0.01$ \\
\hline
\end{tabular}


Table 5. Effect of urea and cassava leaf compost on root weight and size at maturity (142 DAS) of two rice varieties

\begin{tabular}{|c|c|c|c|c|}
\hline \multirow[t]{2}{*}{ Treatments } & \multicolumn{2}{|c|}{ Root weight (g/hill) } & \multirow{2}{*}{$\begin{array}{l}\text { Max. root } \\
\text { length }(\mathrm{c} \mathrm{m})\end{array}$} & \multirow{2}{*}{$\begin{array}{l}\text { Root canopy dia. } \\
\text { (cm) }\end{array}$} \\
\hline & Fresh & Dry & & \\
\hline \multicolumn{5}{|l|}{ Nitrogen $(\mathrm{N})$} \\
\hline Control (C) & $10.68^{C}$ & $9.657^{\mathrm{C}}$ & $21.08^{\mathrm{C}}$ & $9.53^{\mathrm{c}}$ \\
\hline Urea (U) & $14.71^{\mathrm{b}}$ & $12.53^{\mathrm{b}}$ & $23.50^{\mathrm{b}}$ & $9.79^{b}$ \\
\hline $\begin{array}{l}\text { Leaf compost } \\
\text { (LC) }\end{array}$ & $15.60^{\mathrm{a}}$ & $13.61^{\mathrm{a}}$ & $23.71^{\mathrm{a}}$ & $10.57^{\mathrm{a}}$ \\
\hline \multicolumn{5}{|l|}{ Variety (V) } \\
\hline $\begin{array}{l}\text { BRRI dhan29 } \\
\left(V_{1}\right)\end{array}$ & $11.64^{b}$ & $10.46^{b}$ & $21.97^{b}$ & $9.70^{b}$ \\
\hline BINA dhan5 $\left(V_{2}\right)$ & $15.68^{\mathrm{a}}$ & $13.41^{\mathrm{a}}$ & $23.56^{\mathrm{a}}$ & $10.22^{\mathrm{a}}$ \\
\hline \multicolumn{5}{|c|}{ Interaction $(\mathbf{N} \times \mathbf{V})$} \\
\hline $\mathrm{V}_{1} \mathrm{C}$ & $10.5^{\mathrm{e}}$ & $9.2^{\mathrm{e}}$ & $20.3^{\dagger}$ & $9.3^{\dagger}$ \\
\hline $\mathrm{V}_{1} \mathrm{U}$ & $11.6^{\mathrm{d}}$ & $10.7^{\mathrm{cd}}$ & $22.4^{\mathrm{d}}$ & $10.2^{\mathrm{b}}$ \\
\hline $\mathrm{V}_{1} \mathrm{LC}$ & $12.7^{\mathrm{c}}$ & $11.5^{\mathrm{c}}$ & $23.1^{\mathrm{c}}$ & $9.5^{\mathrm{e}}$ \\
\hline $\mathrm{V}_{2} \mathrm{C}$ & $10.8^{\mathrm{e}}$ & $10.0^{\mathrm{de}}$ & $21.8^{\mathrm{e}}$ & $9.7^{\mathrm{d}}$ \\
\hline $\mathrm{V}_{2} \mathrm{U}$ & $17.7^{\mathrm{b}}$ & $14.4^{\mathrm{b}}$ & $23.8^{\mathrm{b}}$ & $10.0^{\mathrm{c}}$ \\
\hline $\mathrm{V}_{2} \mathrm{LCC}$ & $18.4^{\mathrm{a}}$ & $15.6^{\mathrm{a}}$ & $25.0^{\mathrm{a}}$ & $10.9^{\mathrm{a}}$ \\
\hline $\mathrm{N}$ levels $(\mathrm{A})$ & $<0.01$ & $<0.01$ & $<0.01$ & $<0.01$ \\
\hline Variety (B) & $<0.01$ & $<0.01$ & $<0.01$ & $<0.01$ \\
\hline$A \times B$ & $<0.01$ & $<0.01$ & $<0.01$ & $<0.01$ \\
\hline
\end{tabular}

Table 6. Effect of urea and cassava leaf compost on nitrogen uptake in grain, stem and root at maturity (142 DAS) of two rice varieties

\begin{tabular}{|c|c|c|c|}
\hline \multirow[t]{2}{*}{ Treatments } & \multicolumn{3}{|c|}{ Nitrogen uptake (\%) } \\
\hline & Grain & Stem & Root \\
\hline \multicolumn{4}{|l|}{ Nitrogen (N) } \\
\hline Control (C) & $1.22^{\mathrm{c}}$ & $0.53^{\mathrm{C}}$ & $0.44^{\mathrm{c}}$ \\
\hline Urea $(U)$ & $1.84^{\mathrm{a}}$ & $1.20^{\mathrm{a}}$ & $0.75^{\mathrm{b}}$ \\
\hline Leaf compost (LC) & $1.59^{\mathrm{b}}$ & $1.05^{\mathrm{b}}$ & $0.81^{a}$ \\
\hline \multicolumn{4}{|l|}{ Variety (V) } \\
\hline BRRI dhan29( $\left(\mathrm{V}_{1}\right)$ & $1.60^{\mathrm{a}}$ & $1.14^{\mathrm{a}}$ & $0.65^{b}$ \\
\hline BINA dhan5 $\left(V_{2}\right)$ & $1.51^{\mathrm{b}}$ & $0.70^{\mathrm{b}}$ & $0.69^{a}$ \\
\hline \multicolumn{4}{|l|}{ Interaction (V x N) } \\
\hline $\mathrm{V}_{1} \mathrm{C}$ & $1.2^{\mathrm{a}}$ & $0.5^{\mathrm{e}}$ & $0.5^{\mathrm{c}}$ \\
\hline $\mathrm{V}_{1} \mathrm{U}$ & $1.9^{\mathrm{a}}$ & $1.5^{\mathrm{a}}$ & $0.6^{\mathrm{b}}$ \\
\hline $\mathrm{V}_{1} \mathrm{LC}$ & $1.6^{\mathrm{a}}$ & $1.3^{\mathrm{b}}$ & $0.7^{\mathrm{b}}$ \\
\hline $\mathrm{V}_{2} \mathrm{C}$ & $1.1^{\mathrm{a}}$ & $0.5^{\mathrm{e}}$ & $0.3^{d}$ \\
\hline $\mathrm{V}_{2} \mathrm{U}$ & $1.7^{\mathrm{a}}$ & $0.8^{\mathrm{c}}$ & $0.8^{\mathrm{a}}$ \\
\hline $\mathrm{V}_{2} \mathrm{LC}$ & $1.5^{\mathrm{a}}$ & $0.7^{\mathrm{d}}$ & $0.89^{a}$ \\
\hline N levels $(A)$ & $<0.01$ & $<0.01$ & $<0.01$ \\
\hline Variety (B) & $<0.01$ & $<0.01$ & $<0.01$ \\
\hline$A \times B$ & $<0.01$ & $<0.01$ & $<0.01$ \\
\hline
\end{tabular}

Table 7. Chemical properties of cassava leaf compost and soil (before and after harvesting)

\begin{tabular}{lllllll}
\hline Treatments & $\mathbf{p H}$ & $\mathbf{C ~ ( \% )}$ & $\mathbf{N}(\%)$ & $\mathbf{P}(\mathbf{p p m})$ & $\mathbf{K}(\mathbf{p p m})$ & $\mathbf{S}(\mathbf{p p m})$ \\
\hline Cassava Leaf Compost (CLC) & 7.54 & 17.43 & 1.54 & 228.79 & 2567.94 & 189.43 \\
Control soil (before transplanting) & 7.20 & 1.14 & 0.12 & 7.26 & 48.35 & 82.720 \\
Control plot soil (after harvesting) & 7.10 & 1.02 & 0.09 & 4.16 & 40.89 & 68.73 \\
Urea treated soil (after harvesting) & 7.37 & 1.17 & 0.15 & 9.81 & 51.35 & 87.57 \\
CLC treated soil (after harvesting) & 7.42 & 2.35 & 0.58 & 29.07 & 174.56 & 139.48 \\
\hline
\end{tabular}




\section{CONCLUSIONS}

The investigated result of this study showed that cassava leaf compost increased rice growth, yield and nutrient uptake as well as soil fertility status. Composting of cassava leaf and its application along with inorganic fertilizers will reduce the cost of rice farming and improve soil health by increasing $\mathrm{C}$ and $\mathrm{N}$ pool of the soils.

\section{CONSENT}

As per international standard or university standard, patient's written consent has been collected and preserved by the author(s).

\section{ETHICAL APPROVAL}

As per international standard or university standard written ethical approval has been collected and preserved by the author(s).

\section{COMPETING INTERESTS}

Authors have declared that no competing interests exist.

\section{REFERENCES}

1. Das S, Tiwari C, Singh $Y$. Effect of Sesbania green manure and nitrogen fertilizer in rice (Oryza sativa)-wheat (Triticum aestivum) cropping system. Indian J. Agric. Sci. 1995;65(10):780811.

2. Gooding MJ, Davies WP. Foliar urea fertilizations in cereals: A review. Fert. Res. 1992;32:209-222.

3. Adebayo AG, Shokalu AO, Akintoye HA. Effect of compost mixes on vegetative development and fruit. National Horticultural Research Institute, Ibadan; 2011.

4. Hang DT, Preston TR. The effect of simple processing methods of cassava leaves on $\mathrm{HCN}$ content and intake by growing pigs. Livestock Res. Rural Devel. 2005;17(9): 99.

5. Eggum RO. The protein quality of cassava leaves. Brazil. J. Nutr. 1970;24:761-768.

6. Akakpo K. Effects of intercropping systems based on cassava on the soil fertility. In Proc. $12^{\text {th }}$ symp. Intl. Soc. Tropical Root Crops (ISTRC), 'Potential of root crops for food and industrial resources' Sept. Tsukuba, Japan. 2000;10-16.
7. Islam ATMT, Abdullah S, Fakir MSA. Growth and yield in two Cassava Morphotypes. J. Agrofor. Environ. 2007; 1(2):21-24.

8. Fakir MSA, Talukder MHR, Mostafa MG, Rahman MS. Debranching effect on growth and yield in cassava. J. Agrofor. Environ. 2011;5(1):1-5.

9. Akter F. Morphological and growth descriptor for characterization of seven cassava (Manihot esculenta Crantz) accessions. M.S. Thesis. Dept. Crop Bot., Bangladesh Agric. Univ., Mymensingh; 2011.

10. Jannat M. Dry mass content of plant parts, flour extraction and nutrient contents of tuber of cassava accessions. M.S. Thesis, Dept. Crop Bot., Bangladesh Agric. Univ., Mymensingh; 2011.

11. Akhter S. Effect of cassava leaf compost on growth and yield in boro rice. M.S Thesis, Dept. Crop Bot BAU, Mymensingh. 2012;1-33.

12. Jackson ML. Soil chemical analysis. Prentic Hall Inc. Engle Wood Clitts, N.J. USA; 1973.

13. Nelson DW, Sommers LE. Total carbon, organic carbon and organic matter. In Methods of Soil Analysis, Part II (2 ${ }^{\text {nd }}$ edition). Page AL, Miller RH, Keeny DR. Eds., American Soc. Agron., Inc- and Soil Sci. Soc. Soc. Amer., Inc., Madison, Wiscossin. 1982;539-580.

14. Bremner JM. Nitrogen total In: Methods of soil analysis, part 3: Chemical Methods; Sparks, D.L. (ed); Soil Science Society of America: Madison, Wisconsin. 1996;10851121.

15. Olsen SR, Watanabe FS. Test of an ascorbic acid method of determining phosphorus in water and extracts from the soil. Soil Sci. Am. Proc. 1965;26:677-678.

16. Ghosh AB, Bajaj JC, Hasan R, Singh D. Soil and water testing methods: A laboratory manual, Division of Soil Science and Agricultural Chemistry. IARA, New Delhi, India. 1983;1-45.

17. Thomas GW. Exchangeable Cations. Methods of Soil Analysis, Part 2, Chemical and Microbiological Properties. Second Edition. A.L. Page (editor). Agronomy, No. 9, Part 2, American Society of Agronomy, Soil Science Society of America, Madison, WI: 159-165; 1982.

18. Gomez KA, Gomez AA. Statistical procedures for agricultural research. $2^{\text {nd }}$ Edn. John Wiley and Sons, New York, 
Chichester, Brisbane, Toronto, Singapore. 1984;680.

19. Pandey J, Singh RP. Response of newly developed medium duration and high yielding rice genotypes to nitrogen. Indian J. Agron. 1985;30(1):114-116.

20. Furoe RE, Morris RA. Response of transplanted rice to two nitrogen levels and mungbean green manure. Paper presented at the $13^{\text {th }}$ 'Annual Science Meeting' of the CSSP. Cebu City. 1982; 18-20.

21. Balasubramaniyan P. Nitrogen fertilizer for short duration rice. Int. Rice Res. Newsl. 1984;9(5):29.

22. Ghosh BC, Raghavaian CV, Jann MK. Effect of seed rate and nitrogen on growth and yield of direct sown rice (Oryza sativa L.) under intermediate deep water condition. Indian J. Agron. 1991;36:227228.
23. Bhuiyan NI. Crop production trends and need of sustainability in agriculture. The Paper Presented at the Workshop on 'Integrated Nutrient Management for Sustainable Agriculture; Held at Soil Resources Development Institute, Pabna, Bangladesh. 1994;26-28.

24. Diekmann KH, Ottow JG, De Datta SK. Yield and nitrogen response of low land rice (Oryza sativa) to Sesbania rostrata and Aeschynomene afraspera green manure in different marginally productive soils in Philippines. Biol. Fert. Soils. 1996; 21(1-2):103-108.

25. UNDP and FAO. Land resources appraisal of bangladesh for agricultural development. Report No. 2. Agroecological Regions of Bangladesh. United Nations Development Programme, FAO, Rome. 1998;212-221.

(c) 2020 Hawlader et al.; This is an Open Access article distributed under the terms of the Creative Commons Attribution License (http://creativecommons.org/licenses/by/4.0), which permits unrestricted use, distribution, and reproduction in any medium, provided the original work is properly cited. 